\title{
ANALYTIC DESCRIPTION OF THE ELECTRON TEMPERATURE BEHAVIOR IN THE UPPER IONOSPHERE AND PLASMASPHERE.
}

\author{
G. V. Khazanov, A. F. Nagy, T. I. Gombosi \\ Space Physics Research Laboratory, \\ Department of Atmospheric, Oceanic and Space Science, \\ University of Michigan
}

M. A. Koen

Irkutsk Polytechnical Institute

S. J. Cariglia

MIT Haystack Observatory

\begin{abstract}
.
Approximate analytic solutions to the well-known and commonly used time-dependent electron energy balance equation for the upper ionosphere and plasmasphere have been obtained and are discussed. The various potential heating sources for the terrestrial plasmasphere are summarized and the corresponding electron temperatures and related characteristic heating and cooling times are calculated. A comparison between the analytic expressions for the temperature variations and relevant measurements shows excellent agreement.
\end{abstract}

\section{Introduction}

The terrestrial ionosphere/plasmasphere system is a very complex one, but significant progress has been made during the last decade in developing comprehensive theoretical models to describe its behavior [e.g. Schunk et al., 1986; Roble et al., 1987; Guiter and Gombosi, 1990]. These major models are all based on extensive numerical approaches. However, simple analytic solutions are still very useful, because they usually allow a much better understanding of how the system responds to a wide spectrum of relevant parameters. Analytic solutions are also useful in testing numerical model calculations.

In this paper we solve the well known and commonly used energy balance equation for thermal electrons in the upper ionosphere and plasmasphere. We obtain simple analytic expressions for the electron temperature and heat flows into the ionosphere, as well as characteristic heating and cooling times. These questions have been considered before, using numerical models, but our analytic expressions allow a simple and to some degree more helpful insight into the processes controlling the electron temperatures in these regions.

\section{Electron Energy Equation}

The theoretical basis necessary to calculate the electron temperatures in ionosphere and plasmasphere is well-known [e.g., Banks and Kockarts, 1973; Schunk and Nagy, 1978]. The electron energy equation, for altitudes above $200 \mathrm{~km}$, in the absence of field aligned currents, simplifies down to the following:

$$
\frac{3}{2} \mathrm{kn}_{\mathrm{e}} \frac{\partial \mathrm{T}_{\mathrm{e}}}{\partial \mathrm{t}}=\mathrm{B} \frac{\partial}{\partial \mathrm{s}}\left\{\frac{\kappa_{\mathrm{e}} \mathrm{T}_{\mathrm{e}}^{5 / 2}}{\mathrm{~B}} \frac{\partial \mathrm{T}_{\mathrm{e}}}{\partial \mathrm{s}}\right\}-\mathrm{L}_{\mathrm{ei}}-\mathrm{L}_{\mathrm{m}}+\mathrm{Q}_{\mathrm{c}}
$$

Copyright 1992 by the American Geophysical Union.

Paper number 92GL01940

0094-8534/92/92GL-01940\$03.00 where $T_{e}$ is the electron temperature, $B$ is the magnitude of the magnetic field, $s$ is the distance along the magnetic field line, $k$ the Boltzmann constant, $n_{e}$ the electron density, $\kappa_{e}$ is the appropriate constant associated with the thermal conductivity, $\mathrm{L}_{\mathrm{ei}}$ and $\mathrm{L}_{\mathrm{en}}$ are the electron-ion and electron-neutral cooling rates respectively and $Q_{e}$ is electron heating rate.

\section{Sources of Electron Heating}

The main sources of ionospheric electron heating are collisions between superthermal and thermal electrons. Photoelectron production, due to EUV and X-ray solar radiation, peaks between about 100 and $200 \mathrm{~km}$, which is also the altitude range where precipitating electrons deposit most of their energy. These superthermal electrons move along the geomagnetic field lines, changing direction and losing energy via collisions with neutral and charged particles [e.g. Nagy and Banks, 1970; Banks et al., 1974].

Superthermal electrons escaping from the ionosphere experience small-angle scattering, when they move through the plasmasphere, due to Coulomb interactions with the ambient thermal plasma. As a result of this scattering process, some of the electrons are scattered out of the loss cone and become trapped. Sanatani and Hanson [1970] and Nagy and Banks [1970] gave some qualitative discussion of electron trapping and the resulting increase in plasmaspheric heating, but the first attempts of quantitative calculations were those of Gastman [1973], Takahashi [1973] and Lejeune and Worsmer [1976].

Khazanov and Gefan [1982], using the appropriate transport equations for superthermal electrons, obtained the following simple analytic expression for the thermal electron heating rate in the ionosphere and plasmasphere:

$$
Q_{e}(s)=E_{s} q(s)+a_{s} n_{e}(s)
$$

where $E_{s}$ is the mean energy gained locally by the thermal electrons from the superthermal ones, $q(s)$ is the total ionization rate and $a_{s}$ is the strength of nonlocal heating.

There are a number of other, potentially important, processes besides photoelectron heating of the thermal plasma in the plasmasphere. There are numerous indirect indications of such heating processes such as high ion and electron temperatures (at times exceeding 10,000 \% $)$, enhanced heavy ion $\left(\mathrm{O}^{+}\right.$and $\mathrm{O}^{++}$) densities at high altitudes, and electron temperature enhancements with associated SAR arc emissions in the ionosphere [Chandler et al., 1988].

Cole [1965] proposed that Coulomb collisions of the thermal electrons with energetic ring current protons will result in energy deposition in the outer plasmasphere and that this energy will be transported down into the ionosphere via heat conduction, resulting in enhanced temperatures and $6300 \mathrm{~A}$ oxygen airglow emissions, known as SAR ares. Kozyra et al. 
(1987) generalized this mechanism to the case of multicomponent ring current ions, and showed that most of the energy transfer is the result Coulomb collisions with the heavy ring current ions.

Cornwall et al [1971] suggested that ion cyclotron waves generated by the interaction of ring current ions and thermal plasma near the plasmapause is the likely mechanism for the energy transfer. There has been no definitive confirmation of this suggestion to date. In the outer plasmasphere electromagnetic ion cyclotron Alfven waves (A) and fast magnetosonic (FMS) waves can interact efficiently with the thermal electrons via Cherenkov resonance. The rate of this heating was calculated [Khazanov and Chernov, 1988], using quasi-linear theory.

The generation of currents and the associated Joule heating of electrons in the boundary layer, which is formed during the intensification of magnetospheric convection, was discussed by Krymskiy [1990].

Table I shows the range of the expected integrated column heating rates of the plasmasphere by photoelectrons [Khazanov and Gefan, 1982], the ring current [Kozyra et al.,1987], ion-cyclotron waves [Chemov et al., 1990] and Joule heating [Krymskiy, 1990], in units of eV cm-' $\mathrm{cm}^{-1}$.

\section{Electron Temperatures in the Upper Ionosphere}

Let us consider the solution to equation (1) in the topside F region below about $1000 \mathrm{~km}$. The divergence of the magnetic field can be neglected within this small altitude range. Furthermore in the upper ionosphere the time rate of change of the electron temperatures is controlled by the downward heat flow, which has a relatively long time constant. Therefore pseudo steady state conditions are present at these altitudes and the time derivative in the energy equation (1) can be neglected. The two main energy loss processes are Coulomb collisions with the ions and "fine structure" cooling by inelastic collisions with atomic oxygen, the major neutral constituent [cf. Schunk and Nagy, 1978]. Using the expression for the electron heating rate given by (2), the electron energy equation can be written as:

$$
\begin{aligned}
& \frac{\partial}{\partial s}\left\{\kappa_{e} T_{e}^{s / 2} \frac{\partial T_{e}}{\partial s}\right\}-\frac{\gamma_{e i} n_{e}^{2}}{T_{e}^{3 / 2}}\left\{T_{e}-T_{i}\right\}-\frac{\gamma_{e n} n_{n} n_{e}}{T_{n}} \\
& \left\{T_{e}-T_{n}\right\}+E_{s} q+a_{s} n_{e}=0
\end{aligned}
$$

where $\gamma_{\text {ei }}$ and $\gamma_{e n}$ are the appropriate constants for the electronion and electron-neutral loss rates, $n_{n}$ and $T_{n}$ are the neutral densities and temperatures respectively. In writing equation (3) a mathematically simplified form for the fine structure cooling rate was assumed, as indicated. An analytic solution of this equation was obtained, assuming that the altitude variation of the electron density is given by an exponential. The details of the solution were discussed by Khazanov [1979] and will not be repeated here. The limiting expression obtained for the variation of the electron temperature in the upper ionosphere is:

$$
\mathrm{T}_{\mathrm{e}}^{7 / 2}=\mathrm{T}_{\mathrm{n}} \mathrm{T}_{\infty}^{5 / 2}+\frac{7 \mathrm{H}_{\mathrm{s}}^{2}}{2 \mathrm{~K}_{\infty}}\left\{\mathrm{E}_{\mathrm{s}} \mathrm{q}_{\infty}+\mathrm{a}_{\mathrm{s}} \mathrm{n}_{\infty}\right\}-\frac{7 \Phi_{\mathrm{tb}}}{2 \mathrm{~K}_{\infty}}\left\{\mathrm{s}-\mathrm{s}_{\mathrm{o}}\right\}
$$

where $T_{\text {eo }}, q_{\text {eo }}$ and $n_{\mathrm{eo}}$ are the electron temperature, the electron production rate and the electron density at the reference level, $\mathrm{s}_{\mathrm{O}}, \mathrm{H}_{\mathrm{n}}$ is the neutral atomic oxygen scale height and $\Phi_{t b}$ is the electron heat flux at the upper boundary of ionosphere. The effects of electron energy losses to the ions

TABLE I. Integrated Column Heating Rates

\begin{tabular}{lll} 
Photoelectrons & $\Theta_{\mathrm{as}}^{\mathrm{pe}}\left(\mathrm{eV} \mathrm{cm}^{-2} \mathrm{~s}^{-1}\right)$ & $10^{9}-10^{10}$ \\
Ring current & $\Theta_{\mathrm{os}}^{\mathrm{e}}\left(\mathrm{eV} \mathrm{cm}^{-2} \mathrm{~s}^{-1}\right)$ & $10^{8}-10^{10}$ \\
Ion cyclotron & $\Theta_{\mathrm{os}}^{\mathrm{w}}\left(\mathrm{eV} \mathrm{cm}^{-2} \mathrm{~s}^{-1}\right)$ & $10^{9}-10^{11}$ \\
Joule Heating & $\Theta_{\mathrm{oa}}^{\mathrm{J}}\left(\mathrm{eV} \mathrm{cm} \mathrm{cm}^{-2} \mathrm{~s}^{-1}\right)$ & $10^{9}-10^{10}$ \\
\hline
\end{tabular}

TABLE II. Steady State Dayside Electron Temperature

\begin{tabular}{llll}
\hline \hline $\mathrm{Q}_{\mathrm{oa}}\left(\mathrm{eV} \mathrm{cm}^{-2} \mathrm{~s}^{-1}\right)$ & $10^{9}$ & $10^{10}$ & $10^{11}$ \\
$\mathrm{~T}_{\mathrm{ea}} \mathrm{s}(\mathrm{K})$ & 2800 & 5400 & 10500 \\
\hline
\end{tabular}

and neutral show up in the first term on the right hand side of equation (4). When the heating and loss terms become negligible the first term becomes $\mathrm{T}_{\mathrm{eo}}{ }^{7 / 2}$ and the second term vanishes; equation (4) then reduces to the simple and well known expression for electron temperature variations, controlled by thermal conduction only [e.g. Banks and Kockarts, 1973].

The solution for the ionospheric electron temperature, equation (4), depends on the heat flux from the plasmasphere, $\Phi_{\mathrm{tb}}$, which is an unknown parameter. In order to establish the value of this heat flux we must obtain a solution to the plasmaspheric energy balance.

\section{Plasmaspheric Solution}

The electron energy equation in the plasmasphere can be obtained by neglecting the cooling terms in (1), giving:

$$
\frac{3}{2} \mathrm{kn}_{\mathrm{e}} \mathrm{A} \frac{\partial \mathrm{T}_{\mathrm{e}}}{\partial \mathrm{t}}=\frac{\partial}{\partial \mathrm{s}}\left\{\mathrm{A} \kappa_{\mathrm{e}} \mathrm{T}_{\mathrm{e}}^{5 / 2} \frac{\partial \mathrm{T}_{\mathrm{e}}}{\partial \mathrm{s}}\right\}+\mathrm{AQ}_{\mathrm{e}}
$$

where $A(s)$ is the cross section of the magnetic tube. If we assume that the thermal heat flux is zero at the equator [this would be the situation if there is symmetry between the two conjugate hemispheres] we can integrate equation (5), with respect to $s$ from the magnetic equator [apex], $s_{a}$, to an arbitrary position, $s$, along the field line, and obtain the following relation:

$$
\frac{3}{2} \mathrm{k} \frac{\mathrm{n}_{\mathrm{e}}(\mathrm{s}) \mathrm{V}(\mathrm{s})}{\mathrm{A}(\mathrm{s})} \frac{\partial \mathrm{T}_{\mathrm{e}}(\mathrm{s})}{\partial \mathrm{t}}=-\frac{2}{7} \mathrm{k}_{\mathrm{e}} \frac{\partial\left\{\mathrm{T}_{\mathrm{e}}(\mathrm{s})\right\}^{7 / 2}}{\partial \mathrm{s}}+\mathrm{Q}_{\mathrm{e}}(\mathrm{s}) \frac{\mathrm{V}(\mathrm{s})}{\mathrm{A}(\mathrm{s})}
$$

where $V(s)=\int_{s_{2}}^{s} A\left(s^{\prime}\right) d s^{\prime}$ is the volume of the magnetic tube. In order to obtain equation (6) it was assumed that the $s$ variations of $n_{e}, T_{e}$ and $Q_{e}$ are much weaker than that of $A(s)$.

Let us now integrate (6) with respect to $s$ from $s=s_{0}$ to $s$ [recall $s_{0}$ is the lower boundary of the ionospheric solution]:

$$
\frac{3}{2} \mathrm{kn}_{\mathrm{e}}(\mathrm{s}) \mathrm{I}(\mathrm{s}) \frac{\partial \mathrm{T}_{\mathrm{e}}(\mathrm{s})}{\partial \mathrm{t}}+\frac{2}{7} \kappa_{\mathrm{e}}\left\{\mathrm{T}_{\mathrm{e}}^{7 / 2}(\mathrm{~s})-\mathrm{T}_{\mathrm{c}}^{7 / 2}\right\}=\mathrm{Q}_{\mathrm{e}}(\mathrm{s}) \mathrm{I}(\mathrm{s})
$$

where:

$$
\mathrm{I}(\mathrm{s})=\int_{s_{\mathrm{o}}} \frac{\mathrm{V}\left(\mathrm{s}^{\prime}\right)}{\mathrm{A}\left(\mathrm{s}^{\prime}\right)} \mathrm{d} \mathrm{s}^{\prime}
$$

Let us now use equation (7) to write an expression for $T_{e}$ at the equator, $T_{\text {ea }}$, assuming that:

$$
\mathrm{T}_{\infty}^{7 / 2} \ll \mathrm{T}_{e}^{7 / 2}
$$

Given (8) we can rewrite equation (7) as:

where:

$$
\frac{\partial \mathrm{T}_{\mathrm{e}}}{\partial \mathrm{t}}+\alpha \mathrm{T}_{\mathrm{e}}^{7 / 2}-\beta \mathrm{T}_{\mathrm{e}}=0
$$

$$
\alpha=\frac{4 \kappa_{e}}{21 \mathrm{kn}_{\mathrm{ea}} \mathrm{I}_{\mathrm{a}}} ; \quad \beta=\frac{2 \mathrm{Q}_{\mathrm{ea}} \mathrm{I}_{\mathrm{a}}}{3 \mathrm{k} \mathrm{n}_{\mathrm{e}} \mathrm{T}_{\mathrm{e}}^{s}} ; \quad \mathrm{T}_{\mathrm{ea}}^{s}=\left\{\frac{7 \mathrm{Q}_{\mathrm{ea}} \mathrm{I}_{\mathrm{a}}}{2 \mathrm{~K}_{\mathrm{e}}}\right\}^{2 / 7}
$$

and $T_{e a} s$ is the steady state solution of equation (7) for the equator. The a subscripts refer to values of variables at the apex of the field line or in other words at the magnetic equator. Finally in writing equation (9) we substituted $Q_{e a}\left\{T_{e a} / T_{e a} s\right\}$ for Qea. Numerical solutions obtained without this substitution/assumption have indicated that the time taken to reach steady state is not changed to any significant degree; this is so because the second term is more important than the third in establishing the characteristic time of the system. The steady 
TABLE III. Heating Time Constants

\begin{tabular}{cccc}
\hline $\mathrm{L}$ & \multicolumn{1}{c}{2} & 3 & 4 \\
\hline$\tau_{\mathrm{d}}^{1}(\mathrm{sec})$ & $9 \cdot 10^{2}$ & $3.6 \cdot 10^{3}$ & $1.4 \cdot 10^{4}$ \\
$\tau_{\mathrm{d}}^{2}(\mathrm{sec})$ & $1.8 \cdot 10^{2}$ & $7.2 \cdot 10^{2}$ & $2.8 \cdot 10^{3}$ \\
\hline
\end{tabular}

state temperatures, $\mathrm{T}_{\mathrm{ea}} \mathrm{s}$, corresponding to a range of column heating rates, $\Theta_{0 a}$, [where $\Theta_{0 a}=Q_{e a} V_{0} / A_{0}$, and $V_{0}$ and $A_{0}$ are the values of $V$ and $A$ at $s=s_{0}$ ] is given in Table II:

The solution of equation (9) for the case when $\beta \neq 0$ is:

$$
T_{e a}(t)=\frac{T_{a}^{s} T_{e}^{m}}{\left\{T_{e}^{m s / 2}\left[1-e^{-t / r_{d}}\right]+T_{e s}^{s} S / 2 e^{-t / \tau_{d}}\right\}^{2 / 5}}
$$

where $T_{e a}{ }^{m}$ is the initial electron temperature at the equator [e.g. in the morning if solar heating is assumed to be the only heat source] and:

$$
\tau_{\mathrm{d}}=\frac{6 \mathrm{n}_{\mathrm{e}} \mathrm{T}_{\mathrm{es}}^{s}}{5 \mathrm{Q}_{\mathrm{es}}}
$$

is the characteristic heating time of the plasmasphere.

The solution of equation (9) for $\beta=0$ [no heating] can be written as:

$$
T_{e a}(t)=\frac{T_{e s}^{s}}{\left\{1+t / \tau_{n}\right\}^{2 / 5}}
$$

where:

$$
\tau_{\mathrm{n}}=\frac{21 \mathrm{n}_{\mathrm{ea}} \mathrm{I}_{\mathrm{a}}}{4 \kappa_{\mathrm{c}} \mathrm{T}_{\mathrm{as}}^{s / 2}}
$$

is the characteristic cooling time of the plasmasphere. It needs to be restated that these solutions are only valid if inequality (8) is satisfied.

\section{Discussion}

Equation (11) describes the heating of the plasmaspheric electron gas, from its initial value, $T_{e a} m$ to the steady-state value, $T_{e a}$. This rate of transition is characterized by the time constant $\tau_{\mathrm{d}}$. It is convenient to express this parameter in terms of the integrated heating rate of plasmasphere, $\Theta$. Equation (12) for the characteristic time of plasmaspheric can be rewritten as:

$$
\tau_{d}=\frac{6 N}{5 \theta_{0 a t}} T_{e s}^{s}
$$

where $N=n_{e a} V_{0} / A_{0}$, is the approximate total electron content per unit area of the geomagnetic field line from $s_{0}$ to the equator.

Using this plasmaspheric solution we can now calculate the heat flux at the upper boundary of the ionosphere. In order to do this let us write equation (6) for conditions at the upper boundary of the ionosphere:

$$
\Phi_{t b}=-\frac{2 \kappa_{e} A_{b}}{7} \frac{\partial T_{e b}^{7 / 2}}{\partial s}=\frac{3}{2} n_{e b} V_{b} \frac{\partial T_{e b}}{\partial t}-Q_{e b} V_{b}
$$

where the subscript $b$ refers to variables at the assumed boundary between the ionosphere and plasmasphere $[\sim 1000$ $\mathrm{km}]$. Next we make the following reasonable assumptions:

$$
\mathrm{T}_{\mathrm{cb}} \approx \mathrm{T}_{\mathrm{ea}} ; \quad \mathrm{n}_{\mathrm{cb}} \approx \mathrm{n}_{\mathrm{ea}} ; \quad \mathrm{V}_{\mathrm{b}} \approx \mathrm{V}_{\mathrm{o}} ; \quad \Theta_{\mathrm{cb}} \approx \Theta_{\infty}
$$

TABLE IV. Characteristic Heating Times

\begin{tabular}{cccc}
\hline $\mathrm{L}$ & 2 & 3 & 4 \\
\hline$\tau_{\mathrm{n}}(\mathrm{sec})$ & $1.1 \cdot 10^{4}$ & $5.5 \cdot 10^{4}$ & $1.92 \cdot 10^{6}$ \\
$\mathrm{~T}_{\mathrm{a}}^{\mathrm{m}} / \mathrm{T}_{\mathrm{ea}}^{\mathrm{s}}$ & 0.52 & 0.87 & 0.91 \\
\hline
\end{tabular}

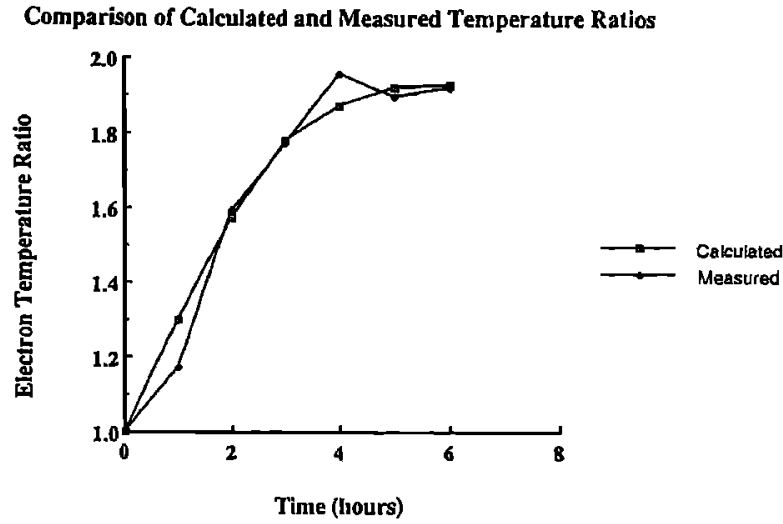

Fig. 1: Comparison of calculated and measured temperature variations for equinox daytime conditions.

Using the approximations given by (17) and using equation (11) to evaluate the time derivative of $T_{\text {ea }}$, we get the following expression for the heat inflow into the ionosphere:

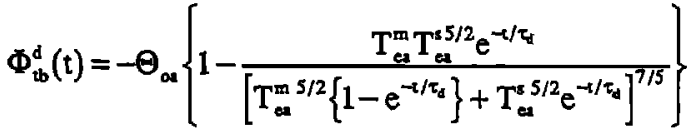

In the limit $\frac{\mathbf{t}}{\tau_{\mathrm{d}}} \gg 1$ (18) leads to:

$$
\Phi_{\mathrm{tb}}^{\mathrm{d}}(\mathrm{t})=-\Theta_{\mathrm{os}}
$$

which is the expected limit; in steady state the energy flow out of the plasmasphere is equal to the volume energy deposition in the plasmasphere.

In a similar manner we can obtain, using (13) and (16), the following expression for the downward heat flow at the ionosphere-plasmasphere boundary for the case of no energy deposition in the plasmasphere [e.g. nighttime conditions when solar heating is the only energy source]:

$$
\Phi_{b b}^{\mathrm{n}}(\mathrm{t})=-\frac{\Theta_{a \mathrm{a}}}{\left\{1+\frac{\mathrm{t}}{\tau_{\mathrm{n}}}\right\}^{7 / 5}}
$$

Note that in the limit of $t / \tau_{n} \rightarrow \infty$ the flux does go to zero, as expected.

The range of plausible heating rates were shown in Table I, ranging from about $10^{8}$ to $10^{11} \mathrm{eV} \mathrm{cm}^{-2} \mathrm{sec}^{-1}$. In Table III the characteristic heating times of the plasmasphere are presented,

Comparison of Calculated and Measured Electron Temperature Ratios

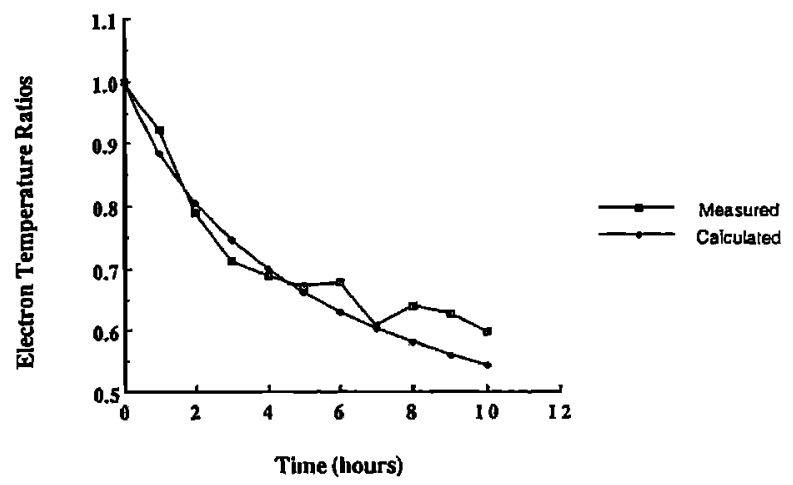

Fig. 2: Comparison of calculated and measured temperature variations for equinox nighttime conditions. 
for different $\mathrm{L}$ values, corresponding to heating rates of $10^{10}$ $\left[\tau_{\mathrm{d}}{ }^{1}\right]$ and $10^{11}\left[\tau_{\mathrm{d}^{2}}\right] \mathrm{eV} \mathrm{cm}^{-2} \mathrm{sec}^{-1}$, respectively. These results, obtained by using equation (12), show that the response times are about one hour or less, a relatively short time compared to the duration of a typical storm.

Let us next examine the cooling of the plasmasphere and the underlying ionosphere. The question of the maintenance of elevated nighttime ionospheric electron temperatures have been of interest since the early 1960's [e.g. Geisler and Bowhill, 1965; Nagy et al., 1968]. It is now well established that at midlatitudes, under magnetically quiet conditions, the energy stored in the plasmasphere is transported down into the ionosphere, via thermal conduction, during the night, elevating the electron temperatures above those of the background neutral gas. Expressions (13) and (20) are important quantitative relations describing the behavior of the electron temperatures at night. They provide a simple tool to evaluate the nightside ionospheric electron temperature variations, and also provide a clear insight into the reasons for the latitude dependence of the temperatures. For example the integrated column electron density in the plasmasphere, $N$, increases with geomagnetic latitude and, under otherwise equal conditions, the rate of drop in plasmaspheric and ionospheric $T_{e}$ decreases. This predicted behavior of nighttime $T_{e}$ agrees well with observations [cf. Schunk and Nagy, 1978]. Table IV shows the calculated characteristic cooling times of the plasmasphere for equinox midnight conditions at different Lshells. The ratios of the plasmaspheric electron temperature at midnight, $T_{e}{ }^{m n}$, to the steady state daytime temperature, $\mathrm{T}_{\mathrm{ea}} \mathrm{s}=4500 \mathrm{~K}$, which is based on photoelectron heating only, are also indicated. These calculations were carried out assuming that $\Theta_{\mathrm{Oa}}=5 \times 10^{9} \mathrm{eV} \mathrm{cm}^{-2} \mathrm{sec}^{-1}$. The corresponding calculated electron temperature at $s=s_{0}$ is $2500 \mathrm{~K}$. [Note that these values satisfy inequality (8)]

Finally we compare our analytic expressions with some observed temperature values. We use the results of radar backscatter observations made at Millstone Hill. The specific data we are using are averages corresponding to $815 \mathrm{~km}$, taken at equinox, during geomagnetically quiet conditions. In Figure 1 we show the measured and calculated [eq. (11)] normalized electron temperature rise. We chose a value of $1760^{\circ} \mathrm{K}$ for $\mathrm{T}_{e} \mathrm{~m}$, based on the data. The curve shown in Figure 1 corresponds to a value of $\mathrm{N} / \Theta_{\mathrm{oa}}$ of $2.15 \times 10^{3} \mathrm{~s} \mathrm{eV}^{-1}$; using a reasonable estimate of $2 \times 10^{13} \mathrm{~cm}^{-2}$ for the column content implies an integrated heating rate of $8 \times 10^{9} \mathrm{eV} \mathrm{cm}^{-2} \mathrm{~s}^{-1}$, which in turn is also of the right magnitude [cf. Schunk and Nagy, 1978]. In Figure 2 we show the normalized electron temperature decrease as measured and as predicted by equation (13). The value adopted from the data for $\mathrm{T}_{\mathrm{ea}} \mathrm{s}$ is $33500 \mathrm{~K}$ and we used $1.1 \times 10^{22} \mathrm{~cm}^{-1}$ for $\mathrm{n}_{\mathrm{ea}} \mathrm{I}_{\mathrm{a}}$. Using this limited data base we found very good agreement between the observed and calculated electron temperature values, giving us added confidence in the validity of our analytic results.

Acknowled gments. This work was supported by the NASA Grants NAGW-1619 and NAG5-1500 as well as NSF Grant ATM-9114409.

\section{References}

Banks, P.M., and Kockarts, Aeronomy, Academic,NewYork, 1973.

Banks, P. M., C. R. Chappell and A. F. Nagy, A new model for the interaction of auroral electrons with the atmosphere: Spectral degradation, backscatter optical emission, and ionization, J. Geophys. Res.. 79, 1459, 1974.

Chandler, M. O., J. U. Kozyra, J. L. Horwitz, R. H.Comfort, and L. C. Brace, Modeling of the thermal plasma in the outer plasmasphere- A magnetospheric heat source,

Modeling Magnetospheric Plasma, edited by T. E. Moore and J. H. Waite, p.101, AGU, Washington, D.C., 1988.
Chernov, A. A., G. V. Khazanov, and S. V. Tanygin, Modeling of electron thermal fluxes into the ionosphere During the excitation of ion cyclotron waves by the ring current, Ann. Geophys., 8, 825, 1990.

Cole, K. D., Stable auroral red arcs, sinks for energy of Dst main phase, J. Geophys. Res., 70, 1689, 1965.

Cornwall, J. M., F. V. Coroniti, and R. M. Thome, Unified theory of SAR-arc formation at the plasmapause, J. Geophys. Res. 76, 4428, 1971.

Gastman, I. J., Theoretical investigation and plasma line measurements of conjugate photoelectrons in the ionosphere, Ph. D. Thesis, U. of Michigan, Ann Arbor, 1973.

Geisler, J. E. and S. A. Bowhill, Exchange of energy between the ionosphere and the protonosphere, J. Atmos. Ter. Phys. 27, 119, 1965.

Guiter, S. M. and T. I. Gombosi, The role of high speed plasma flow in plasmaspheric refilling, J. Geophys. Res. 95, $10427,1990$.

Khazanov G. V., Kinetics of electron component of upper atmosphere plasma (in Russian), Nauka, Moscow, 1979.

Khazanov G. V., and G. D., Gefan, The kinetics of ionosphere-plasmasphere transport of superthermal electrons, Phys. Solariter.. 19.65, 1982.

Khazanov, G. V., and A. A. Chernov, The effect of Alfen and magnetosonic turbulence on the heating of electrons and kinetic coefficients of the plasmasphere, in Ionospheric Investigations (in Russian), Nauka, Vol 44, p. 100, Moscow, 1988.

Kozyra, J. U., E. G., Shelley, R. H. Comfort, L. H. Brace, T. E. Cravens, and A. F. Nagy, The role of ring current $\mathrm{O}^{+}$ in the formation of stable auroral red arcs, J. Geophys. Res., 92, 7487, 1987.

Krymskiy, P. F., Azimuthal currents and heating of the plasma near plasmapause during periods of disturbances, Geomagnetism and Aeronomy, 30, 633, 1990.

Lejeune, J. and F. Wormser, Diffusion of photoelectrons along a field line inside the plasmasphere, J. Geophys. Res. $81,2900,1976$.

Nagy, A. F., and P. M. Banks, Photoelectron fluxes in the ionosphere, J. Geophys. Res. 75, 6260, 1970.

Nagy, A. F., P. Bauer and E. G. Fontheim, Nighttime cooling of the protonosphere, J. Geophys. Res. 73,6259 , 1968.

Rees, M. H. and R. G. Roble, Observation and theory of the formation of stable auroral red arcs, Rev, Geophys. Space Phys., 13, 201, 1975.

Roble, R. G., E. C. Ridley and R. E. Dickinson, On the global mean structure of the thermosphere, J. Geophvs. Res. 92, 8745, 1987.

Sanatani, S. and W. B. Hanson, Plasma temperature in the magnetosphere, J. Geophys. Res. 75 769, 1970.

Schunk, R. W. and A. F. Nagy, Electron temperature in the F Region of the Ionosphere: Theory and Observations, Rev. Geophys. 16, 355, 1978.

Schunk, R. W., J. J. Sojka and M. D. Bowline, Theoretical study of the electron temperature in the high-latitude ionosphere for solar maximum and winter conditions, $\underline{\mathrm{J}}$. Geophys. Res. 91, 12041, 1986.

Takahashi, T., Energy degradation and transport of photoelectrons escaping from the upper ionosphere. Rept. Ionos. and Space Res. Jap.. 27, No.1, 79,1973.

G. V. Khazanov, A. F. Nagy, and T. I. Gombosi, Space Physics Research Laboratory, Department of Atmospheric, Oceanic, and Space Sciences, University of Michigan, Ann Arbor, MI 48109-2143.

M. A. Koen, Irkutsk Polytechnical Institute, Irkutsk, 664074, Russia

S. J. Cariglia, MIT Haystack Observatory, Atmospheric Sciences, Westford, MA 01886. 